\title{
CONTROLE DE TENSÃO NO FLUXO DE POTÊNCIA CONTINUADO - MODELAGENS E EFEITOS NA ESTABILIDADE DE TENSÃO
}

\author{
Carlos Aparecido Ferreira* \\ Vander Menengoy da Costa* \\ kaferredzipmail.com.br \\ vandermcosta@uol.com.br
}

"UFJF, Faculdade de Engenharia, Juiz de Fora, MG, Brasil

\begin{abstract}
This paper proposes to model both the automatic tap control and the secondary voltage control in the continuation power flow problem, as well as, to evaluate the effects in the voltage stability due to the insertion of these control strategies. Maximum and minimum operative limits of each control device are taken into account. Modal analysis results are utilized in order to identify the system critical buses.
\end{abstract}

KEYWORDS: Voltage Stability, Voltage Control, Power Flow.

\section{RESUMO}

Este trabalho propõe modelar o controle automático de tap e o controle secundário de tensão no problema do fluxo de potência continuado, bem como, avaliar os efeitos na estabilidade de tensão decorrentes da inserção destas estratégias de controle. Os limites operativos máximo e mínimo de cada um dos dispositivos de controle são considerados. Resultados de análise modal são utilizados com o objetivo de identificar as barras críticas do sistema.

PALAVRAS-CHAVE: Estabilidade de Tensão, Controle de Tensão, Fluxo de Potência.

Artigo Submetido em 25/03/03

1a. Revisão em 19/03/04;

Aceito sob recomendação do Editor Associado

Prof. Dr. Glauco Taranto

\section{NOMENCLATURA}

$V_{k}^{e s p}, V_{k}^{\text {calc }}=$ tensões especificada e calculada na barra $k$

$\theta_{k}=$ ângulo da tensão na barra $k$

$V_{k}=$ magnitude da tensão na barra $k$

$\gamma=$ carregamento adicional do sistema (p.u.)

$Q_{k}^{e s p}=$ potência reativa especificada na barra $k$

$Q_{k}^{\text {calc }}=$ potência reativa calculada na barra $k$

$Q_{G_{\min }}, Q_{G_{\max }}=$ limites mínimo e máximo de geração reativa

$P_{k}=$ potência ativa líquida injetada na barra $k$

$Q_{k}=$ potência reativa líquida injetada na barra $k$

$Q_{D_{k}}=$ potência reativa demandada na barra $k$

$Q_{G_{k}}=$ potência reativa gerada na barra $k$

$\Delta P_{k}=$ resíduo de potência ativa na barra $k$

$\Delta Q_{k}=$ resíduo de potência reativa na barra $k$

$a=$ Tap do transformador

$\rho=$ passo de variação do parâmetro da continuação

$\alpha_{i, j}=$ fator de participação entre os geradores $i$ e $j$

$\mathrm{BC}=$ barra de tensão controlada

$\mathrm{H}, \mathrm{N}, \mathrm{M}, \mathrm{L}=$ submatrizes da matriz Jacobiana polar 


\section{INTRODUÇÃO}

A estabilidade de tensão pode ser definida como sendo a habilidade do sistema de potência em manter as tensões em níveis aceitáveis, tanto em condições normais de operação, como após um distúrbio (Taylor, 1994). O principal fator que causa a instabilidade de tensão é a inabilidade do sistema elétrico de potência em atender à demanda reativa após um distúrbio (Kundur, 1994).

Os sistemas elétricos de potência estão operando cada vez mais próximos de seus limites. Isso ocorre porque o aumento da demanda de energia elétrica e da complexidade de tais sistemas, não vem sendo acompanhado por investimentos, seja por motivos de ordem econômica, ambiental ou política. Tais fatores têm contribuído para a ocorrência de vários problemas relacionados à estabilidade de tensão.

Situações de instabilidade de tensão como as ocorridas em Baltimore e Washington D.C., EUA, em 1990, e no Japão, em 1987, mostram a necessidade de novas técnicas para análise do problema, como as apresentadas em (Takahata et alii, 1999). No Brasil, após duas ocorrências de colapso de tensão na área de São Paulo, em abril de 1997, foi criada uma Força Tarefa com os objetivos de criar metodologias e definir critérios para estudos de estabilidade de tensão (Martins et alii, 1999). Hoje, as ferramentas computacionais mais amplamente utilizadas são baseadas em levantamentos de curvas PV e VQ, além da utilização da técnica de Análise Modal.

O controle de tensão vem sendo utilizado por várias concessionárias de energia elétrica européias, como é o caso da Electricité de France (Lagonotte et alii , 1989), da Red Eléctrica del Spaña (Sancha et alii, 1996) e da italiana ENEL (Arcidiacono et alii,, 1996). No Brasil, estudos vem sendo feitos sobre a possibilidade de adoção dessa filosofia de controle em determinadas regiões, como no leste de Minas Gerais (Lopes et alii, 2003) e na área do Rio (Taranto et alii, 1999).

Os objetivos deste trabalho são implementar o controle secundário de tensão via injeção de potência reativa feito por geradores e compensadores síncronos, bem como o controle de tensão via variação de taps de transformadores, no problema do fluxo de potência continuado. Tal estratégia de controle apresenta um grande potencial na melhoria do perfil de tensões nas barras e, até mesmo, no aumento das margens de carregamento dos sistemas. Os limites de potência reativa e de taps são considerados, afim de que sejam obtidos resultados mais realísticos .

\section{FLUXO DE POTÊNCIA CONTINUADO}

\subsection{Metodologia Básica}

O fluxo de potência continuado consiste, resumidamente, na obtenção das sucessivas soluções do fluxo de potência através de duas etapas básicas: predição e correção (Ajjapuru e Christy, 1992), conforme apresentado na Figura 1 .

A etapa de predição fornece, através do vetor tangente, a condição inicial para para o processo de obtenção da solução correta. A correção é um processo iterativo resolvido pelo método de Newton Raphson. Nestas etapas são utilizadas as equações básicas de potência expressas em coordenadas polares das tensões nas barras.

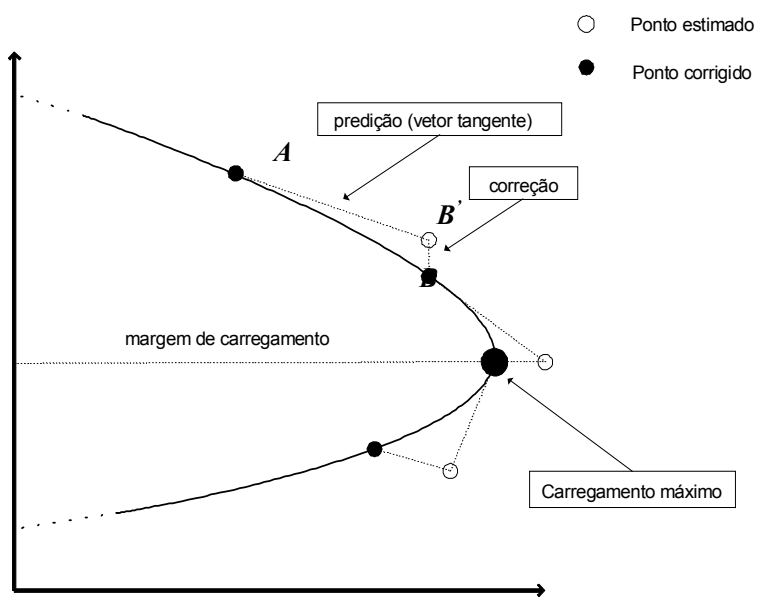

Figura 1: Metodologia de Predição e Correção

A aplicação do método da continuação no problema do fluxo de potência requer a inclusão da nova variável $\gamma$. Assim, considerando-se a topologia mostrada na Figura 2 e o parâmetro da continuação como sendo $\gamma$, os sistemas de equações referentes às etapas de predição e correção são dados por (1) e (2), respectivamente.

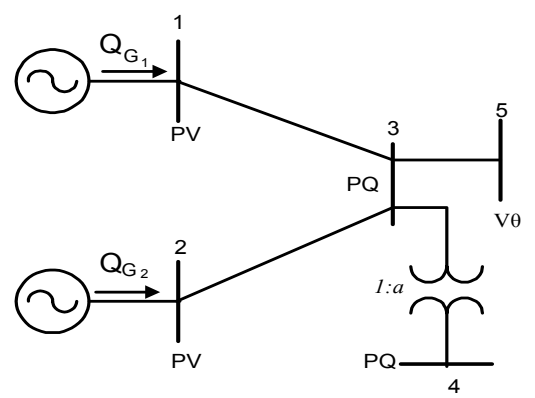

Figura 2: Sistema Teste 
A equação adicional referente ao método da continuação no sistema (1), especifica o valor do parâmetro da continuação para a etapa de correção. Caso o parâmetro da continuação fosse $V_{3}$ ou $V_{4}$, a única modificação seria na posição do elemento não nulo da última linha da matriz Jacobiana. Em (Leite e Da Costa, 2003) são apresentados critérios para a escolha do parâmetro da continuação.

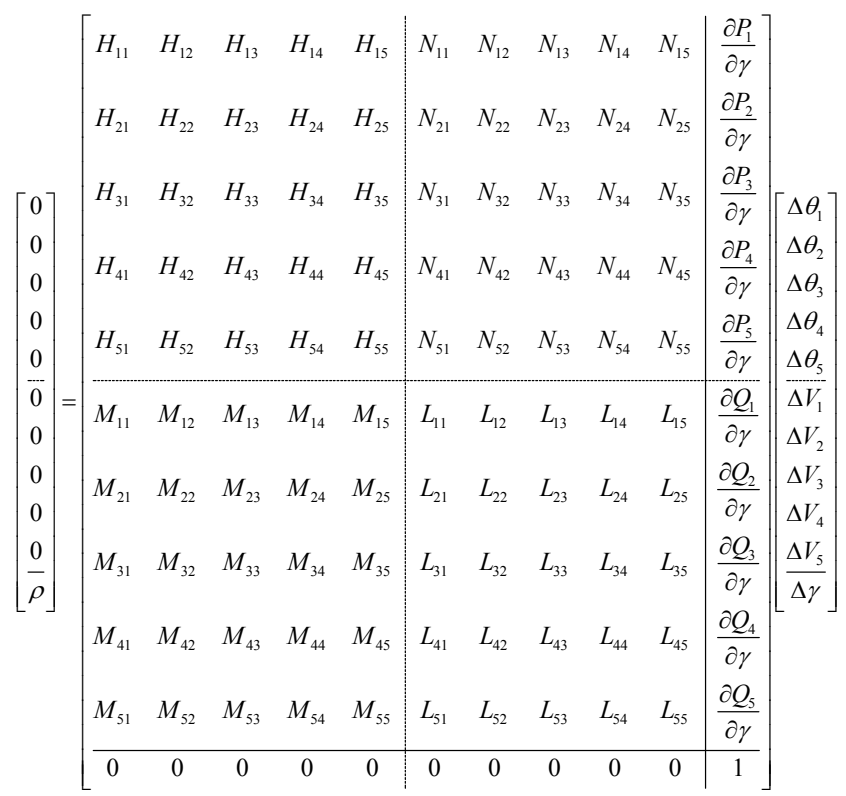

(1)

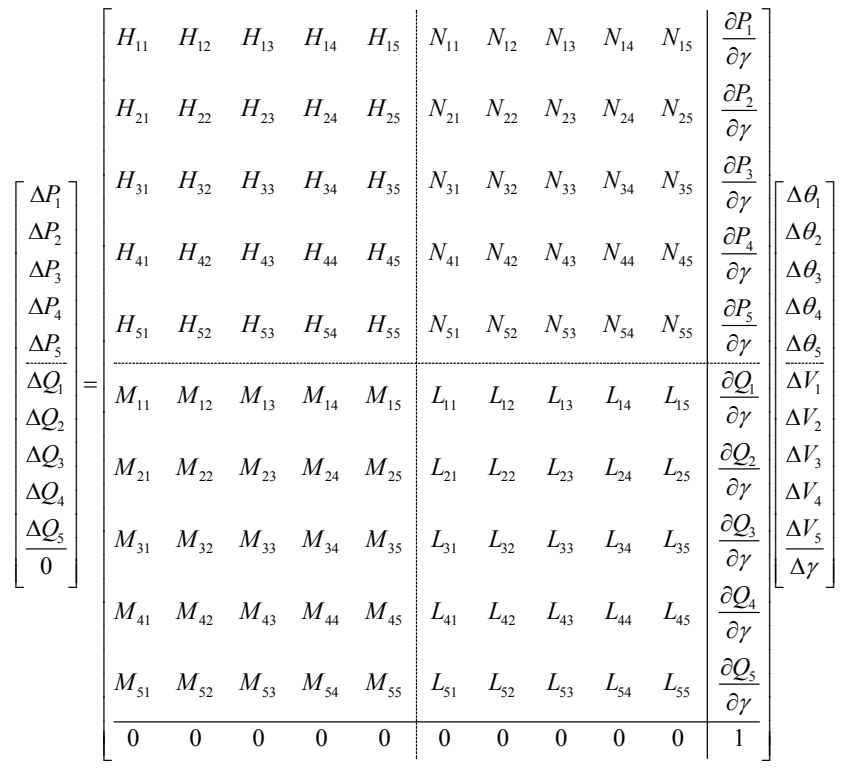

(2)

\subsection{Inclusão do Controle Automático de Tap - CAT}

No CAT, a variação do tap de um transformador é usada para controlar a tensão em uma barra de carga terminal ou remota do transformador. A barra cuja tensão é controlada é do tipo PQV (Monticelli, 1983). A atuação do CAT no processo iterativo ocorre quando o máximo resíduo de potência reativa for menor que uma tolerância préestabelecida .

Considere que o tap do transformador do sistema teste apresentado na Figura 2, controle a tensão na barra 4 .

A equação adicional referente ao CAT é a linearização da equação de tensão referente à barra 4 , do tipo PQV:

$$
V_{4}^{s p}=V_{4}^{\text {calc }}
$$

que resulta em

$$
\Delta V_{4}^{\prime}=\Delta V_{4}
$$

onde

$$
\Delta V_{4}^{\prime}=V_{4}^{s p}-V_{4}^{\text {calc }}
$$

Sendo $\gamma_{0}$ parâmetro da continuação, os sistemas de equações referentes às etapas de estimação e correção são dados por (6) e (7), respectivamente.

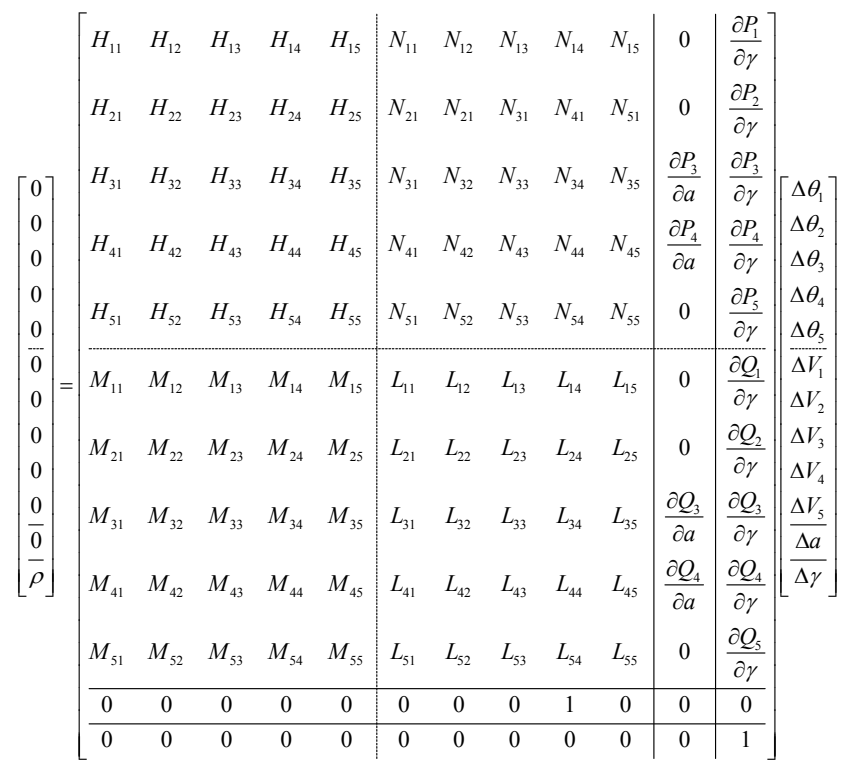




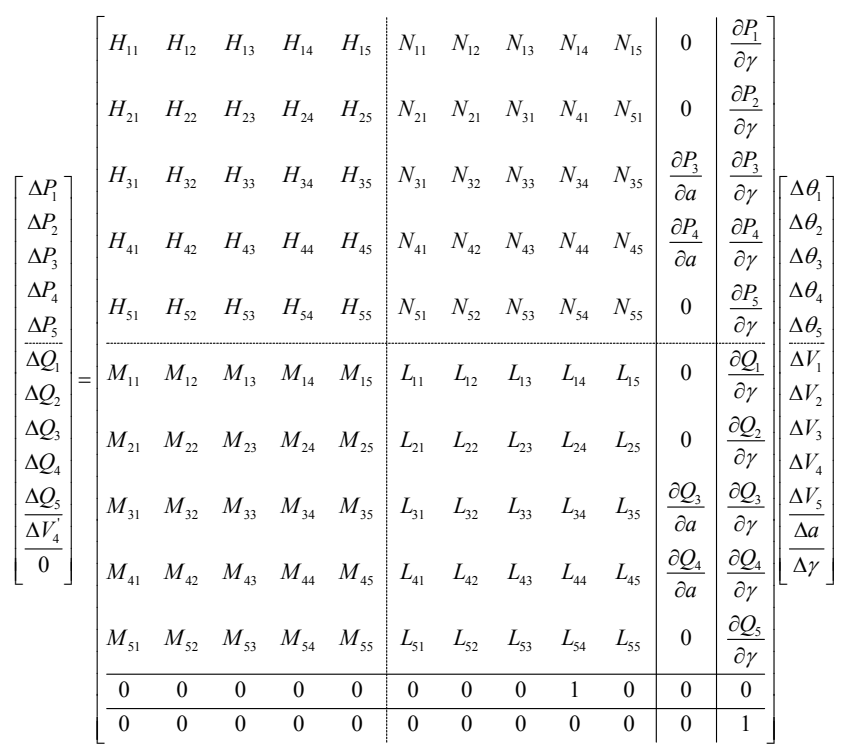

A verificação dos limites de tap dos transformadores, para um dado carregamento $\gamma$, tem início assim que o CAT começar a atuar. Se o limite de algum transformador tiver sido violado, é verificada, a cada etapa subsequente, a possibilidade do mesmo voltar a operar dentro de seus limites (Monticelli, 1983).

\subsection{Inclusão do Controle Secundário de Tensão - CST}

O controle secundário de tensão consiste no ajuste coordenado da tensão em determinadas barras do sistema, denominadas de barras piloto. Este controle pode ser feito através de ajuste individual nos reguladores de tensão dos geradores, compensadores estáticos, compensadores síncronos e ajuste de taps em transformadores. Estes equipamentos controlam a tensão da barra piloto através de fatores de participação. O CST implementado neste trabalho usa suporte de reativos obtido através de geradores/compensadores síncronos em regime permanente, barras do tipo P, para controlar a tensão nas barras piloto, barras do tipo PQV.

\subsubsection{Fatores de Participação}

Seja um sistema com $n_{P}$ e $n_{P Q V}$ barras do tipo P e PQV, respectivamente. Assim, são necessárias $\left(n_{P}-n_{P Q V}\right)$ equações relacionando as potências reativas geradas pelas barras do tipo P. Para dois geradores genéricos $i$ e $j$ tais equações são do tipo:

$$
Q_{G_{i}}-\alpha_{i, j} Q_{G_{j}}=0
$$

A verificação dos limites de potência reativa tem início quando o máximo resíduo de potência reativa for menor que uma tolerância pré-estabelecida. A partir de então, calcula-se, a cada iteração, as potências reativas geradas das barras P e PV, a fim de verificar se está sendo ou não violada a capacidade de geração dos geradores. Se o limite de algum gerador tiver sido violado, verifica-se, a cada etapa subsequente, a possibilidade do mesmo voltar a operar dentro de seus limites (Monticelli, 1983).

Os fatores de participação são definidos neste trabalho segundo uma das formas apresentadas a seguir.

\section{a) Distribuição Igualitária de Potência Reativa}

Os fatores de participação são considerados unitários. Logo, os geradores contribuem com o mesmo valor de potência reativa, independente de sua capacidade. Evidentemente, os geradores com menor capacidade, violarão primeiramente seus limites.

\section{b) Distribuição Proporcional de Potência Reativa}

Cada gerador participante do CST, contribui de acordo com sua capacidade de geração de potência reativa. Para os geradores $i$ e $j$, os fatores de participação são dados pelas relações entre as potências reativas máximas que podem ser supridas pelos geradores.

$$
\alpha_{i, j}=\frac{Q_{G_{\max , i}}}{Q_{G_{\max , j}}}
$$

Segundo essa metodologia todos os geradores participantes do CST atingem seus limites máximos ou mínimos de potência reativa simultaneamente.

\subsubsection{Modelagem proposta}

Considere que no sistema apresentado na Figura 2, além do CAT apresentado no item anterior, controle-se a tensão da barra 3 através das injeções de reativo dos geradores 1 e 2 . Sendo $\gamma$ o parâmetro da continuação, os sistemas de equações referentes às etapas de estimação e correção são dados por (10) e (11), respectivamente. 

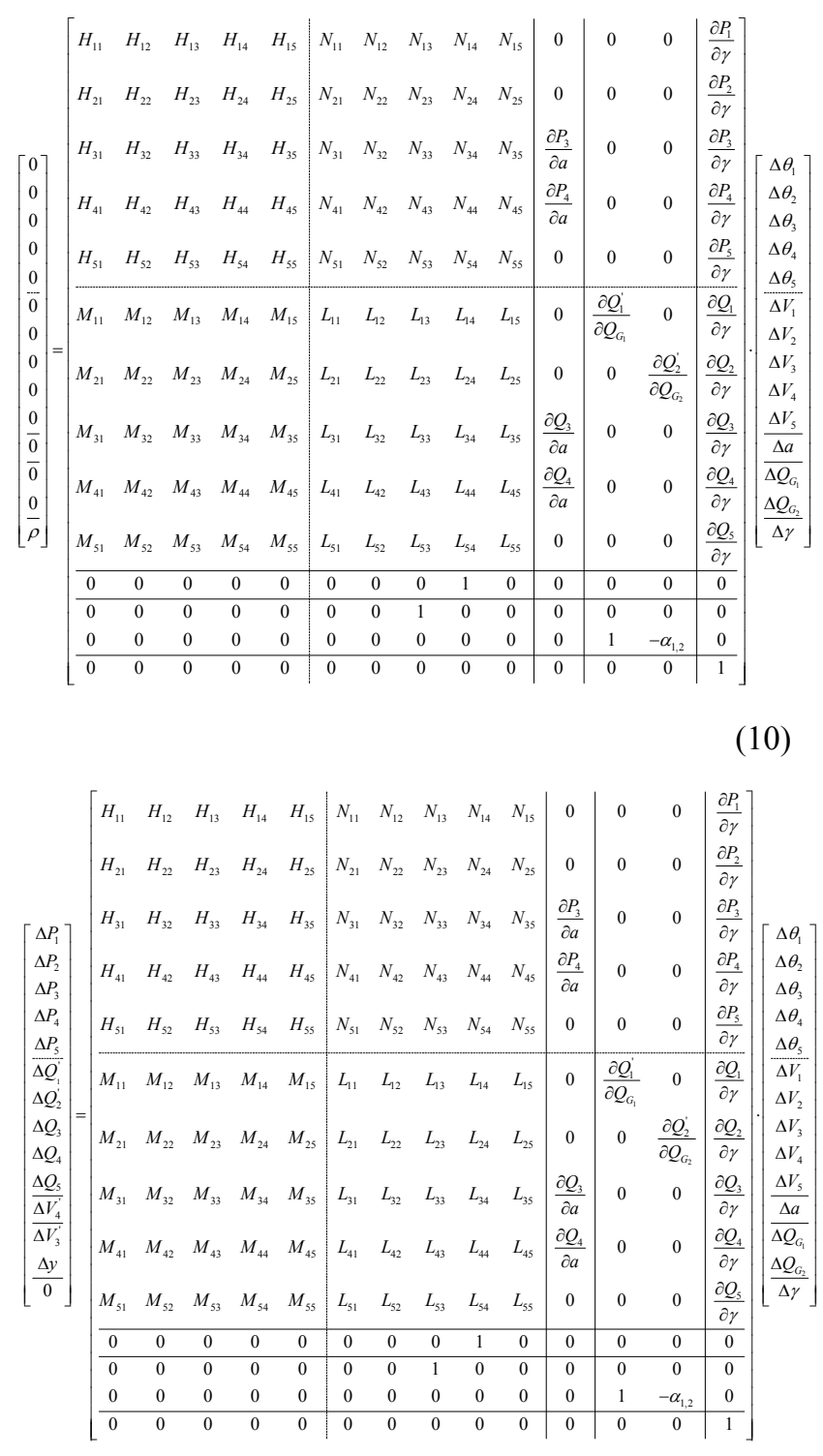

(11)

A primeira equação adicional referente ao CST é a linearização da equação de tensão referente à barra 3 , do tipo PQV:

$$
V_{3}^{s p}=V_{3}^{\text {calc }}
$$

que resulta em

$$
\Delta V_{3}^{\prime}=\Delta V_{3}
$$

onde

$$
\Delta V_{3}^{\prime}=V_{3}^{s p}-V_{3}^{\text {calc }}
$$

A outra equação é a linearização da relação existente entre as potências reativas geradas pelas barras 1 e 2, do tipo P:

$$
Q_{G_{1}}=\alpha_{1,2} Q_{G_{2}}
$$

que resulta em

$$
\Delta y=\Delta Q_{G_{1}}-\alpha_{1,2} \Delta Q_{G_{2}}
$$

onde

$$
\Delta y=-Q_{G_{1}}+\alpha_{1,2} Q_{G_{2}}
$$

Os resíduos $\Delta Q_{1}^{\prime}$ e $\Delta Q_{2}^{\prime}$, referentes às barras do tipo $\mathrm{P}$ são dados por:

$$
\begin{aligned}
& \Delta Q_{1}^{\prime}=Q_{1}^{s p}-Q_{1}^{\text {calc }}=Q_{G_{1}}-Q_{D_{1}}-Q_{1}^{\text {calc }} \\
& \Delta Q_{2}^{\prime}=Q_{2}^{s p}-Q_{2}^{\text {calc }}=Q_{G_{2}}-Q_{D_{2}}-Q_{2}^{\text {calc }}
\end{aligned}
$$

\section{RESULTADOS}

As simulações foram realizadas nos sistemas testes do IEEE (PSTCA, 2004). A tolerância adotada para a convergência do processo iterativo é de $10^{-5}$ p.u..

O modelo de carga utilizado é do tipo potência constante. O carregamento do sistema é feito mantendo-se constante o fator de potência. São considerados os limites de geração das barras PV e a carga adicional é suprida somente pela barra de referência. O limite operacional utilizado para os taps dos transformadores é de 0,9 a 1,1 . O valor utilizado para o passo $\rho$ do parâmetro da continuação é 0,01 .

Resultados de análise modal foram utilizados na determinação de barras piloto. Foram escolhidos transformadores e geradores responsáveis pelos controles nas vizinhanças das barras pilotos, observando-se a topologia dos sistemas testes. Em (Lopes, 2003) são apresentados resultados de análise modal e de análise de sensibilidade corroborando que os dispositivos controladores, na grande maioria das situações, localizamse nas vizinhanças da barra piloto .

\subsection{IEEE- 14 barras}

A análise modal realizada no ponto de colapso, indica que as barras 9 e 4 são críticas, sendo, portanto, escolhidas para ter tensão controlada. A estratégia de controle adotada é, então, que o tap do transformador conectado entre as barras 4 e 9 controle a tensão na barra 9 em 1 p.u., enquanto que a potência reativa gerada nas barras 3 e 8 controle a tensão na barra 4 em 1 p.u., considerando que o fator de participação entre os geradores $\alpha_{3,8}$ seja unitário, ou seja $Q_{G_{3}}=Q_{G_{8}}$. 
As Tabelas 1 e 2 apresentam os resultados da simulação para esta estratégia de controle, considerando o caso base, sendo necessárias 4 iterações para a convergência.

Tabela 1: CAT / IEEE-14 Barras

\begin{tabular}{|c|c|c|c|c|c|c|}
\hline $\boldsymbol{k}$ & $\boldsymbol{m}$ & $\boldsymbol{a}_{\text {inicial }}$ & $\boldsymbol{a}_{\text {final }}$ & $\mathbf{B C}$ & $\begin{array}{c}\mathbf{V}^{\text {esp }} \\
\text { (p.u.) }\end{array}$ & $\begin{array}{c}\mathbf{V}^{\text {calc }} \\
\text { (p.u.) }\end{array}$ \\
\hline 4 & 9 & 0,969 & 0,926 & 9 & 1,0 & 1,0 \\
\hline
\end{tabular}

Tabela 2: CST / IEEE-14 Barras

\begin{tabular}{|c|c|c|c|c|c|c|}
\hline Gerador & $\begin{array}{c}\mathbf{Q}_{\mathrm{G}} \\
\text { (p.u.) }\end{array}$ & $\begin{array}{c}\mathbf{Q}_{\mathrm{G}_{\min }} \\
\text { (p.u.) }\end{array}$ & $\begin{array}{c}\mathbf{Q}_{\mathrm{G}_{\max }} \\
\text { (p.u.) }\end{array}$ & BC & $\begin{array}{c}\mathbf{V}^{\text {esp }} \\
\text { (p.u.) }\end{array}$ & $\begin{array}{c}\mathbf{V}^{\text {calc }} \\
\text { (p.u.) }\end{array}$ \\
\hline 3 & 0,028 & 0 & 0,4 & 4 & 1,0 & 1,0 \\
\hline 8 & 0,028 & $-0,06$ & 0,24 & & & \\
\hline
\end{tabular}

A Figura 3 apresenta o perfil de tensão da barra 9. Com controles atuando, a tensão da barra 9 se mantém no valor especificado até o carregamento adicional atingir 40\%, quando o tap do transformador situado entre as barras 4 e 9 atinge seu valor máximo, conforme pode ser visto na Figura 4.

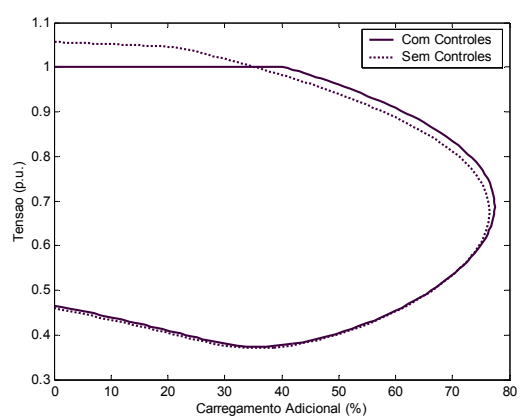

Figura 3 - Perfil de Tensão da Barra 9

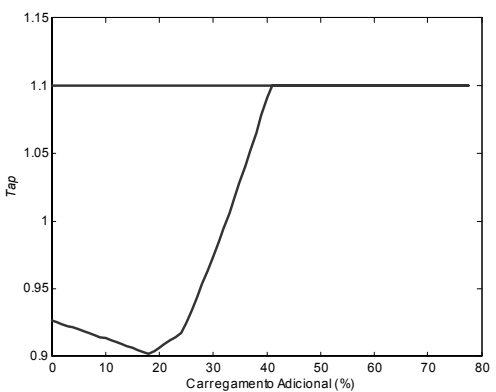

Figura 4 - Tap do Transformador entre as Barras 4 e 9

A Figura 5 apresenta o perfil de tensão da barra 4. Com controles atuando, a tensão da barra 4 se mantém no valor especificado até o carregamento adicional atingir $24 \%$, quando o gerador 3 atinge seu limite máximo de geração de reativo. Como o fator de participação entre os geradores é unitário, o gerador 8 atinge seu limite antes do gerador 3, conforme mostra a Figura 6. Definindo-se o fator de participação segundo a equação (9), os mesmos resultados são obtidos, porém com os dois geradores violando seus limites para um mesmo valor de carregamento.

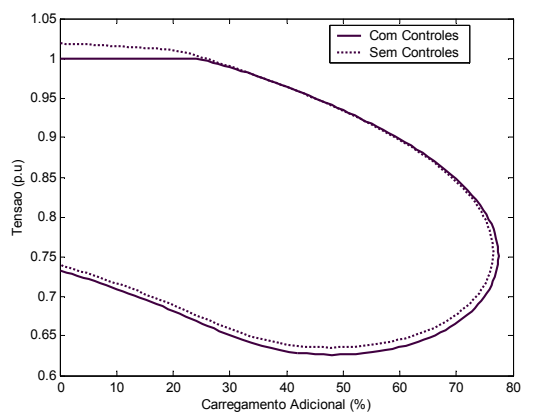

Figura 5 - Perfil de Tensão da Barra 4
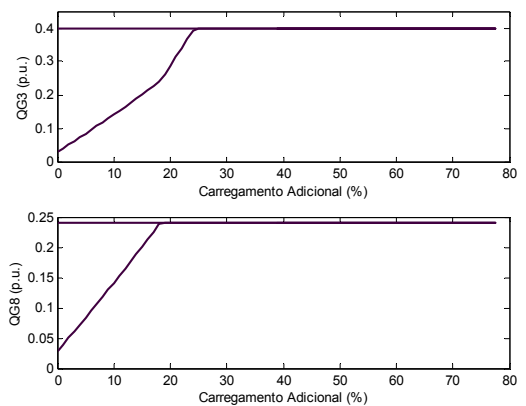

Figura 6 - Potências Reativas Geradas nas Barras 3 e 8

Houve um aumento na margem de carregamento de $76,51 \%$ para 77,43\%. Porém, o principal ganho na aplicação do CST e do CAT é a manutenção do valor da tensão das barras piloto nos valores especificados. Esse é o maior interesse por parte das concessionárias de energia elétrica.

\subsection{IEEE-300 barras}

A análise modal no ponto de colapso, indica que as barras 9033, 9031, 9038 são críticas. Como estas barras estão conectadas à barra 9003, optou-se por efetuar o controle de tensão nesta barra. A outra barra escolhida foi a 526. A estratégia de controle adotada é então que o tap do transformador conectado entre as barras 9001 e 9006 controle a tensão na barra 9003 em 0,983 p.u., enquanto que a potência reativa gerada nas barras 63, 7055 e 7057 controle a tensão na barra 526 em 0,942 p.u., considerando que os fatores de participação entre os geradores $\alpha_{63,7055}$, $\alpha_{7055,7057}$ sejam calculados em termos da equação (9).

As Tabelas 3 e 4 apresentam os resultados da simulação para esta estratégia de controle, considerando o caso base, sendo necessárias 4 iterações para a convergência. 
Tabela 3 : CAT / IEEE-300 Barras

\begin{tabular}{|c|c|c|c|c|c|c|}
\hline $\boldsymbol{k}$ & $\boldsymbol{m}$ & $\boldsymbol{a}_{\text {inicial }}$ & $\boldsymbol{a}_{\text {final }}$ & BC & $\begin{array}{c}\mathbf{V}^{\text {esp }} \\
\text { (p.u.) }\end{array}$ & $\begin{array}{c}\mathbf{V}^{\text {calc }} \\
\text { (p.u.) }\end{array}$ \\
\hline 9001 & 9006 & 0,9668 & 1,0343 & 9003 & 0,983 & 0,983 \\
\hline
\end{tabular}

Tabela 4 : CST / IEEE-300 Barras

\begin{tabular}{|c|c|c|c|c|c|c|}
\hline Gerador & $\begin{array}{c}\mathbf{Q}_{\mathrm{G}} \\
\text { (p.u.) }\end{array}$ & $\mathbf{Q}_{\mathrm{G}_{\min }}$ (p.u.) & $Q_{G_{\max }}$ (p.u.) & BC & $\begin{array}{l}\mathbf{V}^{\text {esp }} \\
\text { (p.u.) }\end{array}$ & $\begin{array}{l}\mathbf{V}^{\text {calc }} \\
\text { (p.u.) }\end{array}$ \\
\hline 63 & 0,207 & $-0,25$ & 0,25 & \multirow{3}{*}{526} & \multirow{3}{*}{0,942} & \multirow{3}{*}{0,942} \\
\hline 7055 & 0,207 & 0 & 0,25 & & & \\
\hline 7057 & 0,745 & $-0,9$ & 0,9 & & & \\
\hline
\end{tabular}

A Figura 7 apresenta o perfil de tensão da barra 9003. A tensão da barra 9003 se mantém no valor especificado até o carregamento adicional atingir $2,11 \%$, quando o tap do transformador situado entre as barras 9001 e 9006 atinge seu valor máximo, conforme pode ser visto na Figura 8.

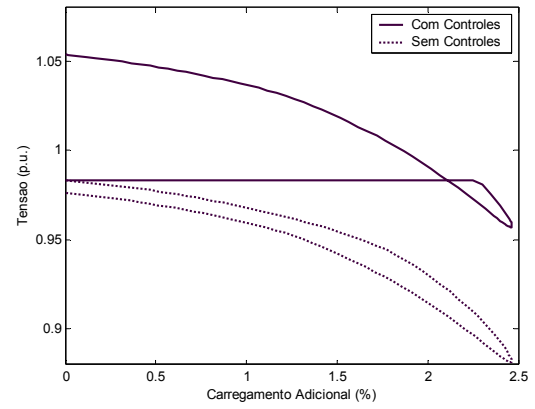

Figura 7 - Perfil de Tensão da Barra 9003

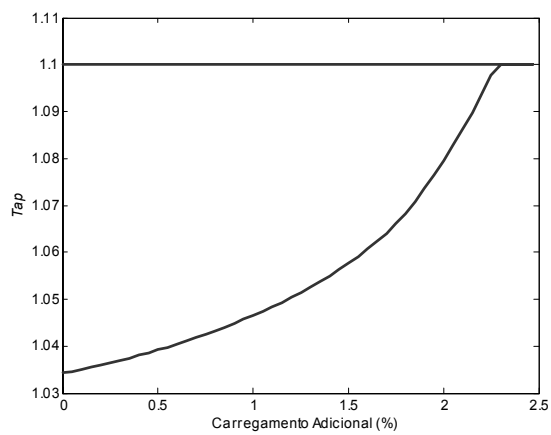

Figura 8 - Tap do Transformador entre as Barras $9001 \mathrm{e}$ 9006

A Figura 9 apresenta o perfil de tensões da barra 9033. Mesmo não sendo controlada diretamente, sua tensão segue a tendência de variação da tensão da barra 9003 . O mesmo comportamento é notado para as tensões das barras 9031 e 9038.

É interessante observar que as curvas mostradas nas Figuras 7 e 9 apresentam um formato não usual quando os controles são ativados, uma vez que as tensões da parte instável das curvas podem atingir valores superiores aos da parte estável, para um mesmo carregamento.

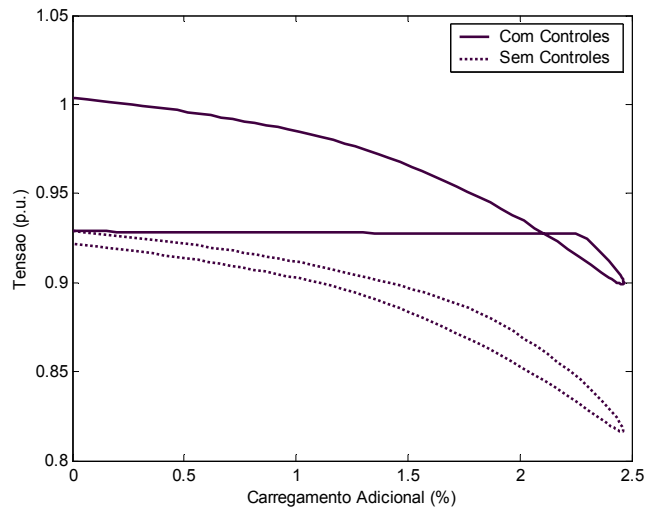

Figura 9 - Perfil de Tensão da Barra 9033

A Figura 10 apresenta o perfil de tensão da barra 526. Com controles atuando, a tensão da barra 526 se mantém no valor especificado até o carregamento adicional atingir $1,35 \%$, quando os geradores atingem seus limites máximos de geração de reativo. Como os fatores de participação foram definidos em função dos limites máximos, os 3 geradores violam seus limites para um mesmo valor de carregamento, conforme pode ser visto na Figura 11.

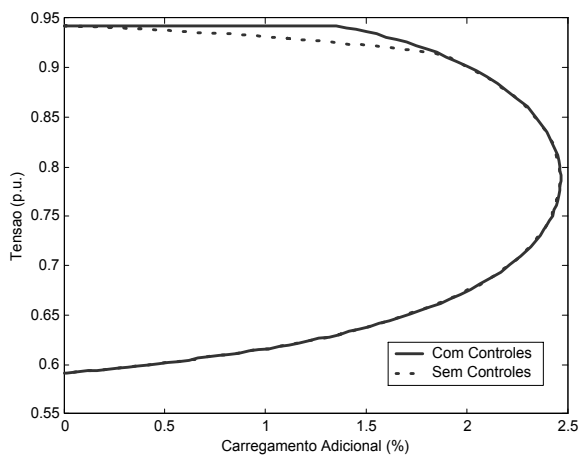

Figura 10 - Perfil de Tensão da Barra 526
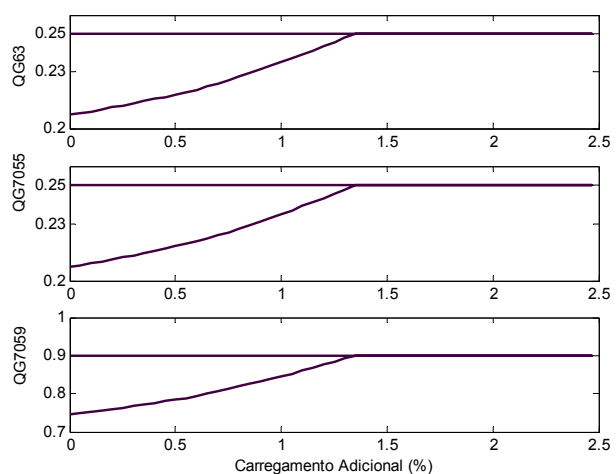

Figura 11 - Gerações de Potência Reativa 


\section{CONCLUSÕES}

Em todos os sistemas analisados, com a utilização de controles, o perfil de tensão se mantém no valor especificado variando-se o carregamento, enquanto há suporte de potência reativa suficiente, no caso do CST, ou enquanto os taps dos transformadores não atingem seus limites, no caso do CAT. Sem os controles, há uma degradação permanente das tensões das barras piloto, conforme verificado.

Os resultados obtidos, alguns dos quais apresentados neste artigo, foram satisfatórios tanto no aspecto do aumento da margem de carregamento, quanto na manutenção das tensões das barras piloto nos valores especificados. Porém, a aplicação prática da filosofia de controle implementada exige estudos aprofundados, pois foram detectados uma série de pontos críticos, que tem sido objeto de várias pesquisas, como a definição das barras piloto, dos geradores e transformadores, dos fatores de participação e das áreas de controle. Além disso, se por um lado tem-se uma maior controlabilidade sobre as variáveis do sistema, por outro, são necessários investimentos adicionais em equipamentos medição e comunicação.

A modelagem no fluxo de potência continuado, dos controles secundário de tensão e de taps de transformadores internamente à matriz Jacobiana, garante a convergência do processo iterativo em um número reduzido de iterações. Tal fato, evidencia a robustez dos modelos apresentados, bem como sua grande aplicabilidade na solução dos problemas atuais inerentes ao controle de tensão. Desta forma, os estudos e modelos desenvolvidos estão em plena consonância com a situação atual de operação dos sistemas elétricos de potência, constituindo-se portanto, como opções no sentido da melhoria da segurança e da estabilidade na operação.

\section{REFERÊNCIAS BIBLIOGRÁFICAS}

Ajjarapu, V., \& C. Christy (1992). The continuation power flow: a tool for steady state voltage stability analysis. IEEE Transactions on Power Systems (February), Vol. 7, No. 1.

Arcidiacono, V., S. Corsi and R. Chinnici (1996). The regional voltage regulator for ENEL'S dispachers. CICRÉ.

Kundur, P. (1994). Power System Stability and Control. Ed McGraw-Hill, Inc. .

Lagonotte, P., J.C. Sabonnadière, J.Y. Leóst. and J.P. Paul (1989). Structural analysis of the electrical system: application to secondary voltage control in France. IEEE Transactions on Power Systems (May), Vol. 4, No.2:479-486.
Leite, L.C.G. \& V.M. Da Costa (2003). Fluxo de potência continuado via formulação de injeção de corrente. Controle \& Automação.

Lopes, T.T., J.L.R. Pereira, V.M. Da Costa e N.S. Gomes (2003). Inclusão do modelo de controle secundário de tensão em um problema de fluxo de potência. XVII Seminário Nacional de Produção e Transmissão de Energia Elétrica, Uberlândia, M.G..

Martins, A.C.B. et alii (1999). Critérios e metodologias no âmbito da força tarefa "Colapso de Tensão" do GTAD/SCEL/GCOI para estudos de estabilidade de tensão nos sistemas interligados Norte/Nordeste, Sul/Sudeste e Norte/Sul Brasileiros. XV Seminário Nacional de Produção e Transmissão de Energia Elétrica, Foz do Iguaçu, P.R..

Monticelli, A.J. (1983). Fluxo de Carga em Redes de Energia Elétrica. 1 ed. Edgard Blucher Ltda, São Paulo, S.P..

PSTCA 2004, Power Systems Test Case Archive, disponível no site http://www.ee.washington. edu/research/pstca.

Sancha, J.L., J.L. Fernández, A. Cortés and J.T. Abarca (1996). Secondary voltage control: analysis, solutions and simulations results for the Spanish transmission system. IEEE Transactions on Power Systems (May), Vol. 11, No.2:630-638.

Takahata, A.Y. et alii (1999). Avaliação de ferramentas computacionais para simulação e análise do fenômeno de instabilidade de tensão. $X V$ Seminário Nacional de Produção e Transmissão de Energia Elétrica, Foz do Iguaçu, P.R..

Taranto, G.N., N. Martins, D.M. Falcão e A.C.B.Martins (1999). Controle secundário de tensão, suas vantagens e limitações, resultados hipotéticos de sua utilização na área do Rio de Janeiro". XV Seminário Nacional de Produção e Transmissão de Energia Elétrica, Foz do Iguaçu, P.R..

Taylor, C. W. (1994). Power System Voltage Stability. Ed McGraw-Hill, Inc.. 